\title{
Aortic Valve Ejection Fraction for Monitoring Heart Contractility in Patients Supported with a Continuous Flow Left Ventricular Assist Device
}

\author{
Viswajith S Vasudevan ${ }^{1}$, Marwan A Simaan ${ }^{2 *}$, Timothy M Maul ${ }^{3}$, Peter D Wearden ${ }^{4}$ \\ ${ }^{1,2}$ Department of Electrical and Computer Engineering, University of Central Florida, Orlando, FL USA \\ ${ }^{3,4}$ Division of Cardiovascular Surgery, Nemours Children's Hospital, Orlando, FL USA
}

Received date: September 04, 2019; Accepted date: October 1, 2019; Publishing date: October 17, 2019

*Corresponding author: Marwan Simaan, Department of Electrical and Computer Engineering, University of Central Florida, Orlando, FL USA, E-mail: simaan@ucf.edu

\begin{abstract}
Ejection Fraction (EF) is an important parameter that is used in the diagnosis of heart failure. It provides a measure of the ability of the heart to pump blood into the circulatory system. For patients implanted with a continuous flow left ventricular assist device (LVAD), the standard definition of EF provides a measure of the combined ability of both the heart to pump blood through the aortic valve and the LVAD to pump blood continuously out of the left ventricle both into the circulatory system. In this paper, we introduce a new concept of EF, labeled Aortic Valve Ejection Fraction (or AVEF) which provides an accurate measure of only the ability of the heart to pump blood through the aortic valve while the LVAD is actively running. We investigate the benefits of AVEF and its relation to the LVAD speed using a well-established mathematical model of an LVAD-assisted left ventricle. We compare the sensitivities of the new AVEF index and the standard EF index to changes in heart contractility over a wide range of after loads. Our results show that AVEF is more sensitive to changes in heart contractility than EF. At a typical LVAD speed of $4,000 \mathrm{RPM}$, a $10 \%$ improvement in heart contractility over time for a patient with congestive heart failure yields $13.20 \%$ increase in AVEF, while the same increase in contractility yields only $7.52 \%$ increase in EF. The AVEF index could provide a reliable, non-invasive mechanism for monitoring improvements in heart contractility for patients implanted with LVAD.
\end{abstract}

Keywords: Ejection Fraction; Aortic Valve Ejection Fraction; Ventricular Elastance; Left Ventricular Assist Device

\section{Introduction}

Cardiac contractility is the intrinsic ability of the heart muscle to generate a force for the left ventricle to contract and pump blood into the circulatory system $[3,13,14,32]$. A reduction in contractility without compensatory changes in other vascular physiologies results in reduced cardiac output, which in turn causes downstream organ dysfunction leading to symptoms of heart failure. Ejection Fraction (EF) is a measure of the ability of the left ventricle to pump blood through the aortic valve, thus acting as a surrogate for the effects of cellular contractility, which is typically not easily measured. EF is defined as

$$
\mathrm{EF}=\frac{\mathrm{SV}}{\mathrm{LVEDV}} \times 100
$$

Where SV = LVEDV - LVESV is the stroke volume, LVEDV is the left ventricular end-diastolic volume and LVESV is the left ventricular end-systolic volume. The numerator in (1) corresponds to the volume of blood ejected through the aortic valve, and the denominator corresponds to the volume of blood available in the ventricle before ejection. Generally, EF in the range of $55 \%$ to $75 \%$ is considered normal while declining EF values less than $50 \%$ indicates progressive heart failure. Measuring EF is an important factor in determining how well the heart is pumping blood and in diagnosing and tracking heart failure.

Heart failure is a disease that affects more than 650,000 Americans every year and in its initial stages is often managed through lifestyle changes and medications [33]. However, as the disease progresses more advanced treatment becomes necessary. In isolated left heart disease, a continuous flow left ventricular assist device (LVAD) is an option for treating patients with advanced heart failure [4, 7, 10, 19, 27, 33]. For patients supported with an LVAD, EF remains an important metric in assessing their heart condition even though it represents the ability of the heart to pump blood while the ventricle is being continuously emptied by the LVAD [11]. LVADs are typically used as a bridge-to-transplantation or destination-therapy. They can also be used as a bridge-to-recovery for a small subset of patients whose heart muscles have the potential to recover with the assistance of an $\operatorname{LVAD}[9,25]$. An important challenge for these patients is in assessing whether the left ventricle is regaining its ability to pump while supported by the LVAD.

Recent studies have identified LVAD parameters to assess contractility of the heart $[2,17]$. The measurement of cardiac performance as an assessment of heart contractility has also been studied extensively $[6,15,16,18]$. For patients without an LVAD, one standard measure to assess contractility is EF as defined in (1) $[1,22]$. The numerator and denominator of (1) can be determined using the left ventricular volume (LVV) curve. However, for LVAD patients the left ventricle is not sufficiently preloaded due to the flow in the LVAD, causing a change in the shape of the LVV curve. Any measure of EF with an LVAD implanted will consequently reflect the combined ability of both the heart and the LVAD to 
pump blood, hence not providing an accurate measure of cardiac performance. Therefore, a new index that takes into account the presence of the LVAD and gauges the sole ability of the heart to pump blood through the aortic valve despite alteration in preload and afterload may be beneficial. When measured periodically, this index should be able to determine if the heart's pumping ability is improving while the LVAD is still implanted.

In this paper, we propose a definition for this EF index, which we will refer to as the Aortic Valve Ejection Fraction (or AVEF). We investigate the feasibility of using AVEF, in comparison to $\mathrm{EF}$, to monitor improvements in heart contractility that might occur while the LVAD is implanted. The lack of availability of animal or human heart contractility and LVAD hemodynamic data currently limits our ability to test AVEF on in-vivo data. As a proof of concept, we utilize a standard validated left ventricleLVAD mathematical model based on a generic axial continuous flow rotary pump. This mathematical model is dynamic, timevarying and nonlinear and has been extensively used in prior LVAD studies $[24,5,30]$. It consists of six coupled time-varying differential equations that represent the relationships between the hemodynamic variables of the left ventricle, including the left ventricular pressure (LVP), the left atrial pressure (LAP), the arterial pressure (AP), the aortic pressure (AoP), the total aortic flow $\left(Q_{T}\right)$ and pump flow $\left(Q_{\mathrm{p}}\right)$. The model provides enough complexity to give an accurate representation of the left ventricle and LVAD functions. The model is driven by the elastance function $\mathrm{E}(\mathrm{t})$ of the left ventricle, defined over one cardiac cycle and the rotational speed in RPM of the LVAD pump.

\section{Definition and Characterization of AVEF}

In the presence of a continuous flow LVAD, the pump continuously unloads the left ventricle, while blood flows through the aortic valve only during the ejection phase of the cardiac cycle. Figure 1 shows two typical LVV curves for the same patient with congestive heart failure ( $\mathrm{CHF}$ ), one for a ventricle without LVAD and the other for the same ventricle with an LVAD running at 4,000 RPM. For the patient without LVAD, the numerator of EF in 1 corresponds to the difference between the volumes at points $\mathrm{D}$ and $\mathrm{C}$ (or A and $\mathrm{B}$ ), while the denominator corresponds to the volume at point $\mathrm{D}$.

If the standard EF (1) is used for an LVAD patient (as is currently the practice), then points $\mathrm{D}^{\prime}$ and $\mathrm{C}^{\prime}$ are used instead of $\mathrm{D}$ and $\mathrm{C}$ in calculating the numerator and denominator of (1). The resulting measure of $\mathrm{EF}$ will no longer reflect the ability of the heart to pump through the aortic valve, but instead it will now reflect the combined ability of both, heart and LVAD, to pump into the circulatory system.

Due to the continuous flow through the LVAD, the contraction and relaxation phases are not isovolumic and hence are difficult to distinguish on the LVV for the ventricle with LVAD. The difference between the volumes at points $\mathrm{A}^{\prime}$ and B' represents the sum of blood ejected through the aortic valve and withdrawn by the LVAD, and points C' and D' represent the beginning and end of the filling phase. Thus, in order to account for the effect of the LVAD on LVV, instead of considering the end diastolic and systolic volumes, the denominator in our new definition should be the volume of blood available in the ventricle immediately before ejection. Thus, we define the Aortic Valve Ejection Fraction, AVEF, as:

$$
\mathrm{AVEF}=\frac{\mathrm{V}_{\mathrm{AV}}}{\mathrm{LVVBE}} \times 100
$$

Where $\mathrm{V}_{\mathrm{AV}}$ is the volume of blood ejected only through the aortic valve and LVVBE is the left ventricular volume immediately before the beginning of ejection. In Figure $1, V_{A V}$ is the difference in volume between points A' and B' adjusted by the amount of blood withdrawn by the LVAD and LVVBE corresponds to the volume at $A^{\prime}$ which is the blood volume in the ventricle immediately at the time when the aortic valve opens.

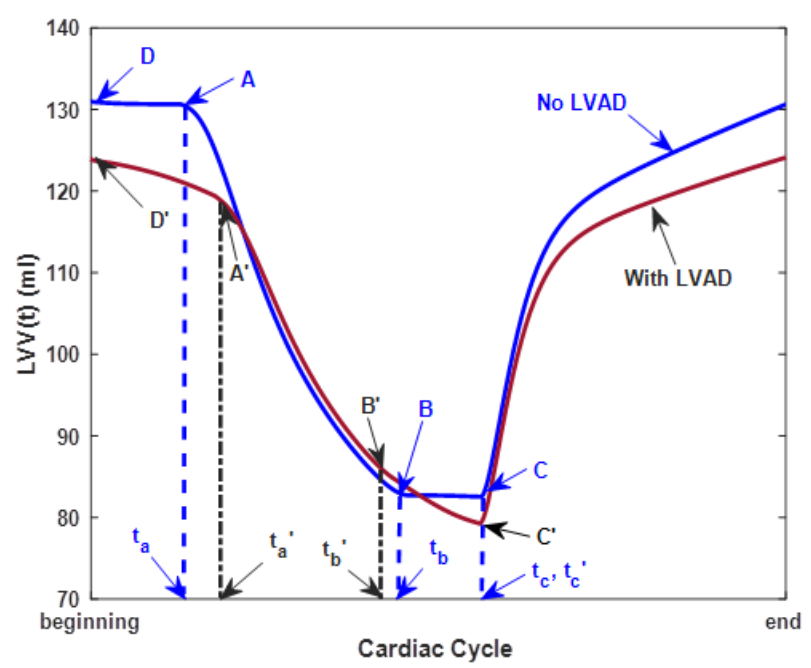

Figure 1: Plot of LVV for a patient without LVAD (blue) and with LVAD (brown) over one cardiac cycle. The times $t_{a}$ and $t_{b}$ represent the beginning and end of the ejection phase and $t_{c}$ represents the beginning of the filling phase. Variables with prime notation correspond to the patient with LVAD

Unlike EF, where the numerator and denominator can be easily determined from the LVV curve, the numerator and denominator of AVEF cannot be easily determined from the LVV curve because points A' and B' (the end of diastole and systole) are not easily identifiable on the curve. To be able to identify these points accurately, the effect of the pump needs to be removed from the LVV with LVAD. Figure 2 shows a plot of $V_{\text {LVAD }}$, the volume of blood withdrawn from the left ventricle by the LVAD during the same cardiac cycle, and Figure 3 shows the LVV plot of Figure 1 adjusted by removing $\mathrm{V}_{\text {LVAD }}$. With this adjustment, the beginning and end of the ejection phase become easily identifiable as point $A^{\prime}$, the point at which LVV starts decreasing and point B' when LVV becomes constant again. Thus, points A' and B' correspond to the instants of the opening and closing of the aortic valve as shown in Figure 1. Points B' in Figures 1 and 3 do not correspond, due to the volume of blood that was withdrawn by the pump while the aortic valve was open between $\mathrm{t}_{\mathrm{a}}$ and $\mathrm{t}_{\mathrm{b}}{ }_{\mathrm{b}}(13.4 \mathrm{ml}$ in this case). 
Additionally, point $\mathrm{C}^{\prime}$ in Figure 3 represents the beginning of the filling phase, thus identifying the relaxation phase. Removing the effects of the pump alters the LVV curve in the filling phase, as can be seen in Figure 3. This will not affect the AVEF index since the filling phase does not play a role during ejection. Thus, from the $\mathrm{LVV}$ plot shown in Figure 3, $\mathrm{V}_{\mathrm{AV}}=21 \mathrm{ml}$ represents the difference in volume between points $A^{\prime}$ and $B^{\prime}$, and $L V V B E=119 \mathrm{ml}$ is the corresponding volume at point $\mathrm{A}^{\prime}$. This yields a calculated AVEF = $(21 / 119) \times 100=18 \%$. For this patient, calculating EF using points $\mathrm{D}^{\prime}$ and $\mathrm{C}^{\prime}$ yields $\mathrm{EF}=((124-79) / 124) \times 100=36 \%$. If this patient did not have an LVAD, his EF would be determined using points $\mathrm{D}$ and $\mathrm{C}$ as $\mathrm{EF}=((130-83) / 130) \times 100=36 \%$. For the patient with LVAD, the difference between $\mathrm{AVEF}=18 \%$ and $\mathrm{EF}=36 \%$ is because in addition to the flow through the aortic valve, $\mathrm{EF}$ includes blood flow through the LVAD while AVEF does not. It should be pointed out that for a patient without LVAD, AVEF will be the same as EF.

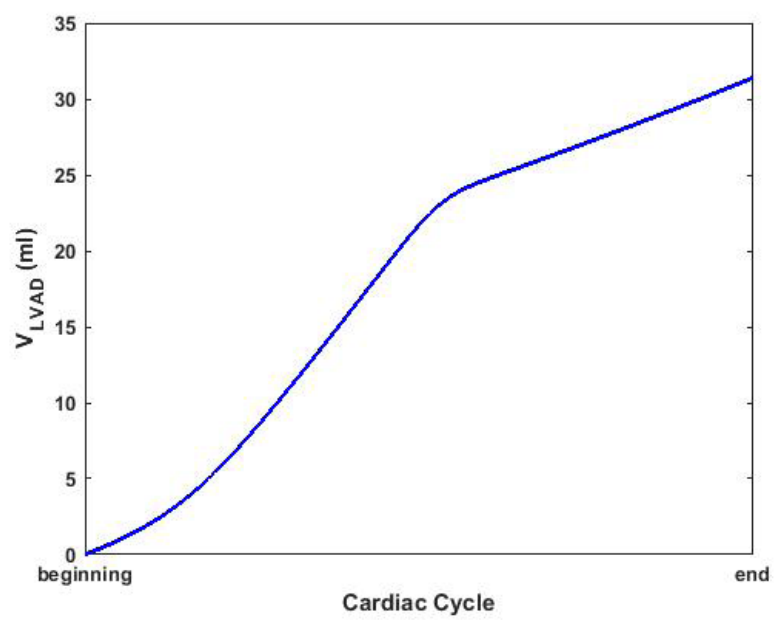

Figure 2: Plot of $V_{L V A D}$, the volume of blood withdrawn by the LVAD over one cardiac cycle

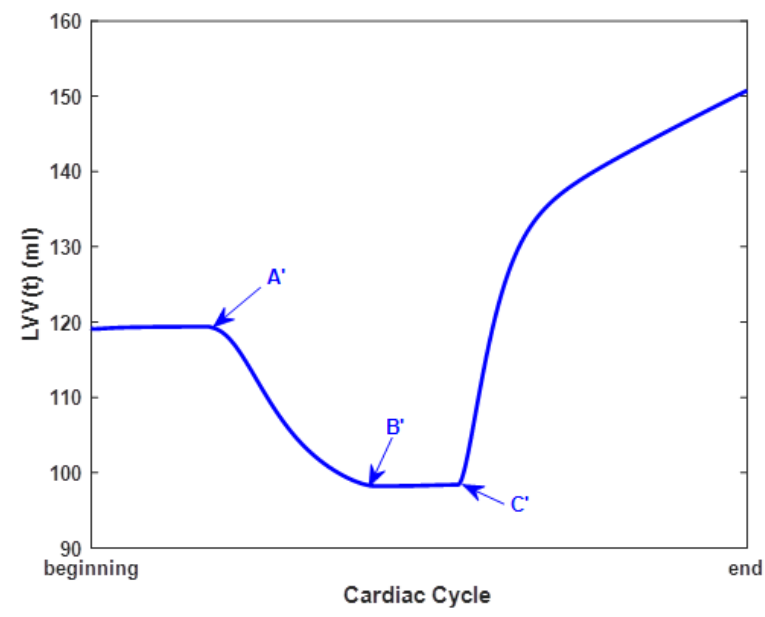

Figure 3: Plot of LVV for a patient with LVAD after the effect of the LVAD flow is removed

\section{Characterization of AVEF and EF as a Function of LVAD Speed}

Contractility of the heart muscle is generally measured in terms of its elastance, which represents the change in pressure per unit volume $[26,28]$. An important measure of the elastance function is its maximum value, $\mathrm{E}_{\max }$, which usually occurs within the ejection cycle. A correlation between $\mathrm{E}_{\max }$ and $\mathrm{EF}$ to establish the degrees of CHF as defined to follow the New York Heart Association (NYHA) classification is noted in Table $1[12,29]$. Figure 1 was obtained using our model for a CHF patient with $\mathrm{E}_{\max }=1.0 \mathrm{mmHg} / \mathrm{ml}$ without LVAD and with an LVAD set at 4,000 RPM (LVAD flow rate of $2.35 \mathrm{~L} / \mathrm{min}$ ).

Table 1: Map of NYHA Classification and Condition of Heart Failure

\begin{tabular}{|c|c|c|c|}
\hline Condition of Heart & EF (\%) & $\begin{array}{c}\mathbf{E}_{\max } \\
(\mathbf{m m H g} / \mathbf{m l})\end{array}$ & $\begin{array}{c}\text { NYHA } \\
\text { Class }\end{array}$ \\
\hline Normal & $50-70$ & $>2.0$ & - \\
\hline Mild Dysfunction & $40-49$ & $1.25-2.0$ & I \\
\hline Moderate Dysfunction & $30-39$ & $0.8-1.25$ & II \\
\hline Severe Dysfunction & $<30$ & $<0.8$ & III - IV \\
\hline
\end{tabular}

An important distinction between EF and AVEF is that AVEF depends on the LVAD rotational speed. Thus, in order to test the effectiveness of AVEF we examined the LVV curve with and without LVAD data for different degrees of CHF and different rotational speeds of the LVAD. We simulated the hemodynamics of an LVAD patient with $\mathrm{E}_{\max }=1.0 \mathrm{mmHg} / \mathrm{ml}$ and calculated AVEF for a range of speeds from 2,000 to 10,000 RPM. The resulting plot, Figure 4 shows that AVEF decreases with increasing speed and confirms that the contribution of the left ventricle to the total flow decreases as the contribution of the LVAD increases. At $6,700 \mathrm{RPM}$, the aortic valve closes permanently and AVEF $=0 \%$

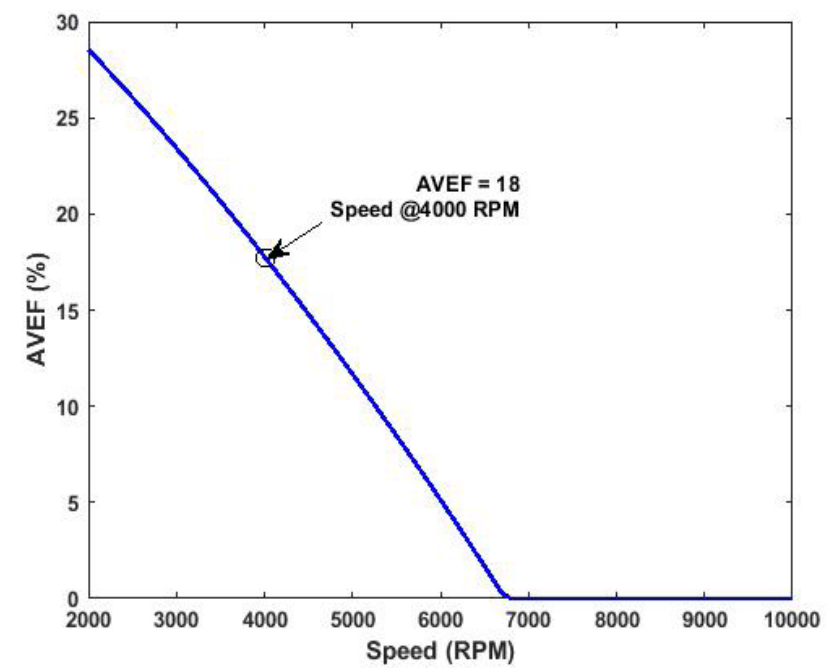

Figure 4: AVEF versus LVAD Rotational Speed 
reflecting the fact that the total flow at this and larger speeds are solely through the LVAD with no flow through the aortic valve. The specific case that was discussed earlier in the LVV of Figure 1 at 4,000 RPM is indicated in Figure 4 showing an AVEF $=18 \%$. Table 2 provides a comparison of AVEF and EF for the same LVAD patient considered in Figure 1 whose unassisted EF is 36\%. This table shows that AVEF decreases rapidly while EF changes very little with increasing speed up to a speed of 6,700 RPM when the aortic valve closes. For speed larger than 6,700 RPM, AVEF $=0$ while EF increases rapidly and reaches a value of $49 \%$ at a speed $10,000 \mathrm{RPM}$. The increased distortion in the EF values at large LVAD speeds, makes EF an unreliable index for estimating the correct patient's ejection fraction when the aortic valve is closed at large LVAD speeds.

Table 2: Comparison of AVEF and EF at Different LVAD Speeds for an LVAD Patient Whose Unassisted EF is 36\%

\begin{tabular}{|c|c|c|}
\hline Speed (RPM) & AVEF (\%) & EF (\%) \\
\hline 2,000 & 28 & 37 \\
\hline 3,000 & 23 & 36 \\
\hline 4,000 & 18 & 36 \\
\hline 5,000 & 12 & 35 \\
\hline 6,000 & 5 & 35 \\
\hline 7,000 & 0 & 35 \\
\hline 8,000 & 0 & 39 \\
\hline 9,000 & 0 & 44 \\
\hline 10,000 & 0 & 49 \\
\hline
\end{tabular}

\section{Sensitivity of AVEF to Changes in Heart Contractility}

As mentioned earlier, the current practice is to rely on the EF index to monitor possible improvements in heart contractility for patients supported with LVAD. The question is; how does AVEF compare to EF in assessing improvements in heart contractility in LVAD patients? While the absolute value of these indices may not be of much interest, it is their sensitivity to small changes in contractility that provides the necessary information about the condition of the heart in an LVAD patient. In many cases, marginal improvements in heart contractility may be of interest and these can only be detected if the index can provide sufficient sensitivity to these changes to make the detection possible.

Since AVEF depends on the LVAD speed, its usefulness will be most effective if monitored at a specific speed setting. This does not disadvantage AVEF since the current practice is to keep the speed setting fixed in outpatient LVAD control. For a fixed speed setting, it is important to first assess if an increase in AVEF over a period of time can be attributed to an increase in contractility over the same period. Figure 5a shows plots of AVEF versus Emax for LVAD speeds in the range 2,000 to 10,000 RPM. As seen in the previous section, AVEF is equal to 0 when the aortic valve is permanently closed. Thus, an important property of AVEF is that its value does incorporate the status of the aortic valve at each speed setting. Furthermore, there are no irregularities in the AVEF profile over the range of $\mathrm{E}_{\max }$. These plots show that at any fixed speed setting, there is a clear positive correlation between $\mathrm{AVEF}$ and $\mathrm{E}_{\max }$.

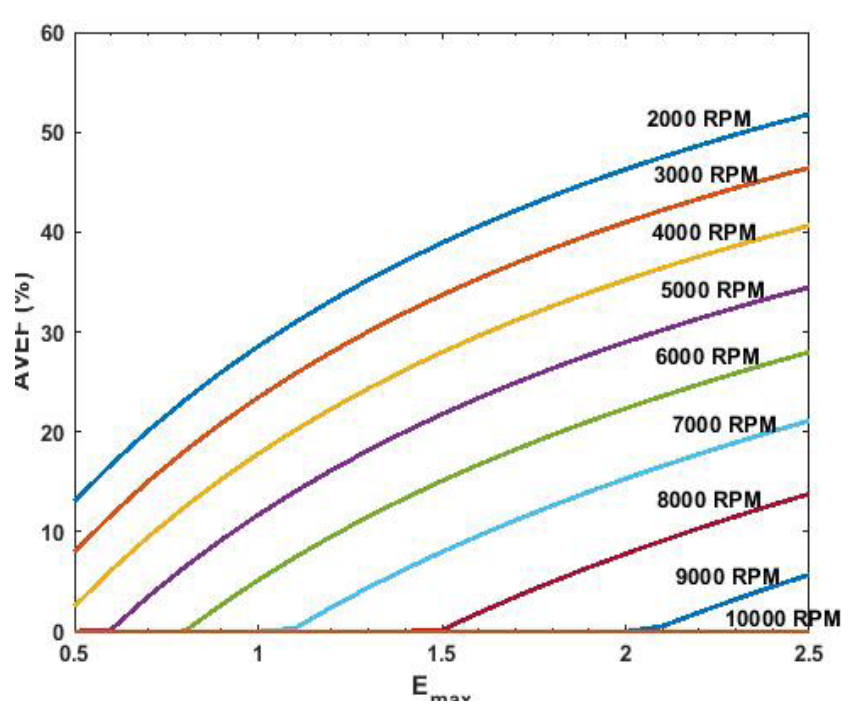

Figure 5a: AVEF versus $E_{\text {max }}$ parametrized for speeds in the range 2,000 to $10,000 \mathrm{RPM}$

Next, we will examine the sensitivity of AVEF to variations in $\mathrm{E}_{\max }$ at a fixed LVAD speed over a wide range of patient physiological conditions. The patient's afterload is altered using the systemic vascular resistance SVR. A value of SVR=1.0 $\mathrm{mmHg}$-s/ml was taken as the base value representing normal afterload. At a fixed LVAD speed of 4,000 RPM, Figure 5b shows a bar chart plot of AVEF for values of SVR ranging from 0.8 to 1.2 $\mathrm{mmHg}$-s/ml. For each value of afterload, $\mathrm{E}_{\max }$ was increased from 0.5 to $2.5 \mathrm{mmHg} / \mathrm{ml}$. This Figure illustrates how AVEF varies with after load and heart contractility. For a fixed afterload, AVEF

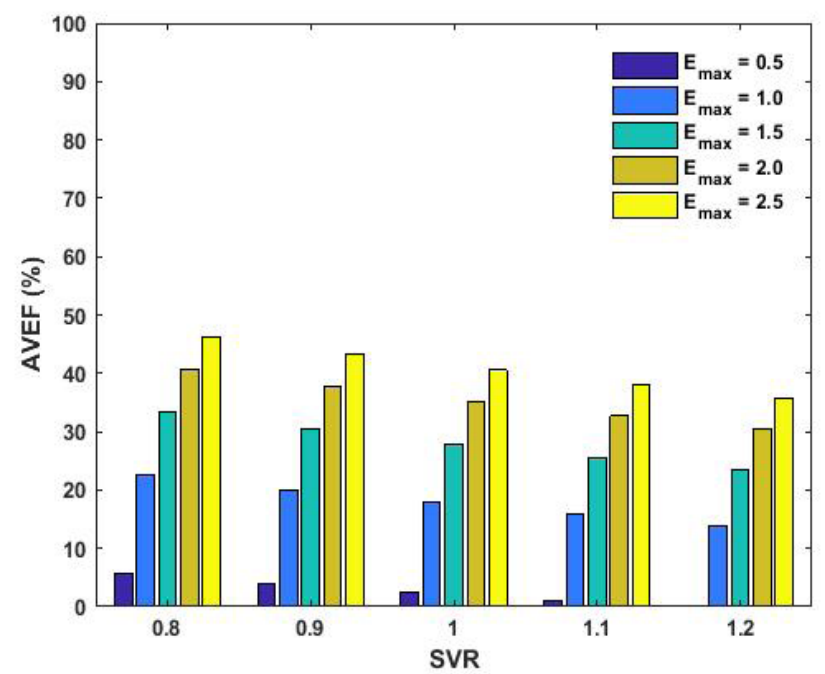

Figure 5b: AVEF versus SVR (afterload) for different CHF conditions characterized by $\mathrm{E}_{\text {max }}$ 
increases with increasing $E_{\max }$ and for a fixed $E_{\text {max }}$ AVEF decreases with increasing afterload. Knowledge of the afterload, however, would still be required if the exact amount of increase in Emax is needed. As an illustration, at an after load of SVR $=1.0$ a change in $\mathrm{E}_{\max }$ from 1.0 to 1.5 (50\% increase) yields a change in AVEF from 18 to 28 (a $56 \%$ increase). Similarly, for an $E_{\max }=1.5$, a change in SVR from 1.0 to 1.1 (10\% increase) yields a change in AVEF from 28 to 26 (7\% decrease). This high sensitivity of AVEF to changes in Emax for a wide range of physiological conditions is largely due to the fact that the flow through the aortic valve, which AVEF measures, carried with it a high percentage of information about contractility of the left ventricle.

\section{Why is AVEF more Reliable than EF to Correlate with Contractility?}

In order to appreciate the differences in sensitivity and characteristics between AVEF and EF, we reproduced the results of Figures $5 \mathrm{a}$ and $5 \mathrm{~b}$ when EF is used instead of AVEF for the same LVAD patient. Figures $6 \mathrm{a}$ and $6 \mathrm{~b}$ show plots of EF versus $\mathrm{E}_{\max }$ for the same range of LVAD speeds and afterloads considered for AVEF in Figures 5a and 5b. A close examination of Figure 6a shows a much more disorganized behavior of EF versus speed (this is also apparent in Table 2) than Figure 5a. Additionally, the behavior for $\mathrm{E}_{\max }<1.5$ is completely different that when $\mathrm{E}_{\max }>1.5$. Figure $6 \mathrm{~b}$ shows that EF is less sensitive than AVEF to variations in heart contractility. For the same illustration considered above in the case of AVEF, the $50 \%$ increase in $\mathrm{E}_{\max }(1.0$ to 1.5$)$ resulted in an increase in EF from 36 to 48 (33\% increase) and the $10 \%$ increase in SVR resulted in a decrease in EF from 48 to 46 (4\% decrease).

Table 3 provides a comparison of the sensitivities of AVEF and EF due to changes in $E_{\max }$ for a patient with an LVAD speed set at 4,000 RPM and average after load (SVR=1). As an illustration, a $10 \%$ improvement in $\mathrm{E}_{\max }$ results in $13.20 \%$ increase in $\mathrm{AVEF}$

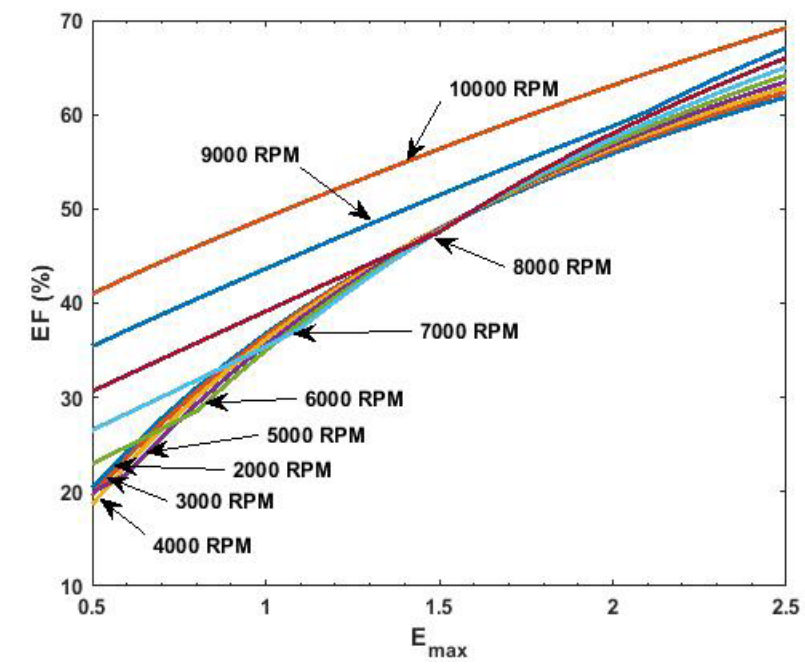

Figure 6a: $\mathrm{EF}$ versus $\mathrm{E}_{\max }$ parametrized for speeds in the range 2,000 to 10,000 RPM

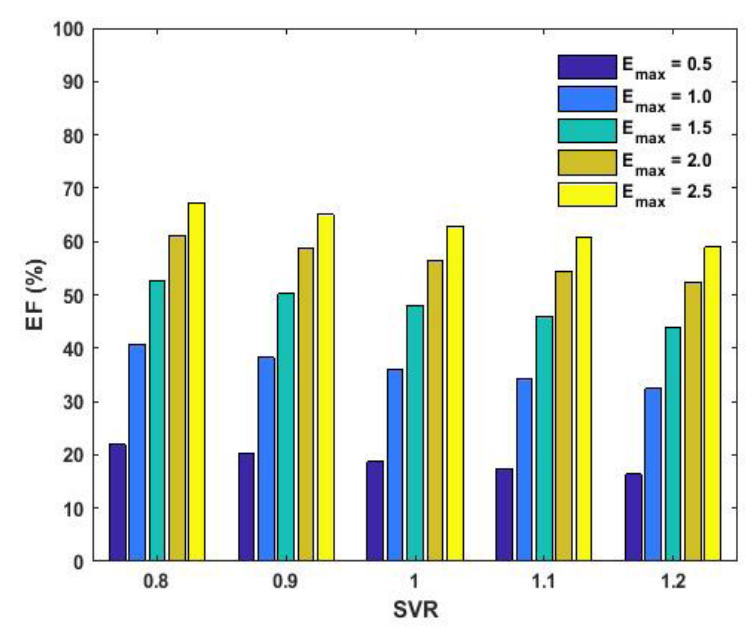

Figure 6b: EF versus SVR (afterload) for different CHF conditions characterized by $\mathrm{E}_{\max }$

\begin{tabular}{|c|c|c|}
\hline \multicolumn{3}{|c|}{ Table 3: Sensitivity of AVEF and EF to Changes in $\mathrm{E}_{\max }$} \\
\hline $\mathbf{\Delta E}_{\max }$ & $\mathbf{\Delta A V E F}$ & $\mathbf{\Delta E F}$ \\
\hline $1 \%$ & $1.40 \%$ & $0.77 \%$ \\
\hline $2 \%$ & $2.74 \%$ & $1.55 \%$ \\
\hline $5 \%$ & $6.77 \%$ & $3.82 \%$ \\
\hline $10 \%$ & $13.20 \%$ & $7.52 \%$ \\
\hline $15 \%$ & $19.45 \%$ & $11.08 \%$ \\
\hline $20 \%$ & $25.44 \%$ & $14.50 \%$ \\
\hline $25 \%$ & $31.22 \%$ & $17.81 \%$ \\
\hline
\end{tabular}

and a $7.52 \%$ increase in EF. In general, our research indicates that for a wide range of $\mathrm{E}_{\max }$ values, LVAD speeds and afterloads, AVEF is approximately twice as sensitive to changes in Emax as EF. It should be pointed out that for the range of Emax from 0.5 to 2.5, values of AVEF are consistently lower than values of EF as can be seen when plots of Figure 5a and compared to Figure 6a. This difference corresponds to the positive bias introduced in EF due to the flow in the LVAD. As noted in Table 2, this bias becomes larger at larger speeds. One of the main disadvantages of this bias is that it amplifies the effects of measurement errors.

Finally, it may be interesting to examine the difference between AVEF and EF from a hemodynamic perspective. One of the main problems of using EF in LVAD patients is that the linearity of the ESPVR, which is characteristic in patients without LVAD, is now altered due to the presence of the LVAD. According to the literature, the LVAD alters the linearity of ESPVR which affects the linear relationship between the Left Ventricular Volume (LVV) and Left Ventricular Pressure (LVP) whose ratio corresponds to the ventricular elastance [18]. Thus, to get a true measure of elastance, the linear relationship between LVV and LVP, and hence the linearity in the ESPVR, needs to be restored in 
LVAD patients. This restoration is accomplished by removing the LVAD flow from the LVV curve as is done in AVEF.

\section{Map Based on Frank-Starling Curves for Correlating AVEF and Contractility}

In this section, we establish a one-to-one relationship between change in AVEF and $\mathrm{E}_{\text {max }^{\prime}}$ and examine the corresponding Frank-Starling curve using components of AVEF as defined in (2). The Frank-Starling curve establishes a relationship between SV and end diastolic volume [8,31]. This curve is used to monitor improvements in cardiac function in patients with CHF [20]. For patients with a continuous flow LVAD, SV is $\mathrm{V}_{\mathrm{AV}}$, and the enddiastolic volume is LVVBE. A heart with improving contractility if supported with an LVAD should show an increase in $V_{A V}$ and a decrease in LVVBE resulting in increasing AVEF.
Figure 7 shows plots of $V_{A V}$ versus LVVBE with varying contractility $\left(\mathrm{E}_{\text {max }}\right)$, afterload (SVR) and LVAD speed (that does not cause the aortic valve to close permanently). Each colored group refers to the family of Frank-Starling plots for the same $\mathrm{E}_{\max }$ value. The contractility increases from the family of curves shown in green to the family of curves shown in blue. Within the family of curves, the speed of LVAD that each curve represents is specified next to the curves in green, and the curves are indicated by a different curve pattern. In each of these curves, the value of SVR changes, as indicated. Operating points of interest are combined together by solid black lines, representing the same after load conditions, same LVAD speed, and different $\mathrm{E}_{\max }$ values. All operating points shown in the plot represent an after load identified by an SVR = 1.0.

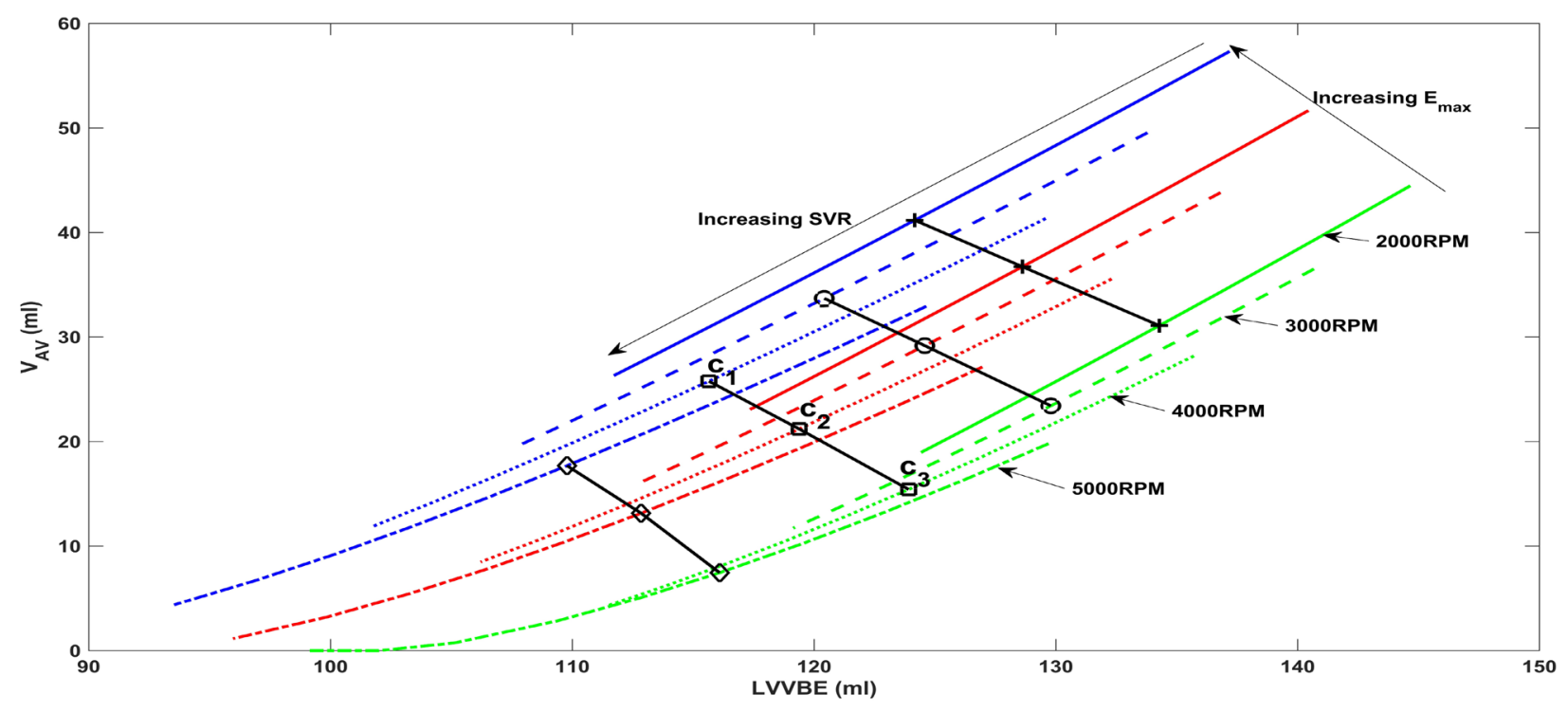

Figure 7: Generic Map of Frank - Starling Curves $\left(\mathrm{V}_{A V}\right.$ versus LVVBE) for different $\mathrm{E}_{\max }$ with changing speeds and SVR

This map confirms that an increase in AVEF may be due to an increase in Emax. For example, for a specific LVAD speed (say 4,000 $\mathrm{RPM})$, as one moves from point $\mathrm{c}_{3}$ to point $\mathrm{c}_{1}$, AVEF increases $\left(\mathrm{V}_{\mathrm{AV}}\right.$ increases while LVVBE decreases) corresponding to increasing $E_{\max }$. The same can be observed for the other sets of operating points as marked on the map. Such an analysis is possible to be made when any of the three parameters considered is changing. To know for certain that an increase in AVEF is only due to $\mathrm{E}_{\text {max' }}$ further analysis of the model has to be carried out which is beyond the scope of this paper. An interesting observation that arises out of this generic map and which would be the next step in our research is to consider other scenarios such as changing speed (ramp test) and constant monitoring (outpatient setting).

To summarize, from a clinical perspective, the strong positive correlation between AVEF and $\mathrm{E}_{\text {max }}$ is very important in that it does suggest that periodic measurements of this index in bridge-to-recovery patients, may provide crucial information in assessing the recovery condition of the patient's heart and the proper timing for LVAD removal.

\section{Conclusion}

The standard definition of EF, while an appropriate measure of the contractility of the left ventricle, is not suitable when used for patients with an LVAD because of the continuous unloading of the ventricle by the LVAD. For patients implanted with an LVAD, the standard definition of EF does not provide an accurate measure of heart contractility since it measures the combined ability of the heart and the LVAD to pump blood into the circulatory system. In this paper, we proposed to consider the ratio of the volume of blood ejected only through the aortic valve to the volume of blood available in the left ventricle immediately before the beginning of the ejection phase as an appropriate measure for the heart contractility. This new aortic valve ejection fraction index, or AVEF, takes into account the fact that the LVAD is continuously emptying the left ventricle and removes this LVAD flow in determining its ejection fraction.

The dependence of AVEF on LVAD speed necessitates that any monitoring of changes in the heart condition using this index must 
be performed at a specific fixed speed setting. We showed that for a fixed speed setting there is a positive correlation between AVEF and heart contractility as measured through its elastance function $\mathrm{E}(\mathrm{t})$. We compared the effectiveness of this index against the standard EF index in assessing changes in contractility of the heart. Our results show that for a wide range of afterload, a realistic increase in heart contractility over time produces a significant and measurable percent increase in AVEF but a much smaller percent increase in EF. Using a Frank-Starling analysis, we established a relationship between changes in AVEF and ventricular contractility. The problem of accurately estimating the LVAD flow in a clinical environment so that it can be subtracted from the LVV curve to determine AVEF remains a challenge that needs to be overcome. Pending data from additional clinical studies, our conclusion indicates that monitoring the heart contractility of an LVAD patient might be effectively accomplished by monitoring the patient's AVEF.

\section{References}

1. Armstrong WF, Ryan T. 2012. Feigenbaum's echocardiography: Lippincott Williams \& Wilkins.

2. Capoccia M. 2016. Mechanical circulatory support for advanced heart failure: are we about to witness a new "gold standard"? J Cardiovasc Dev Dis. 2016;3(4):35. doi: 10.3390/jcdd3040035

3. de Tombe PP. Altered contractile function in heart failure. Cardiovasc Res. 1998;37(2):367-380.

4. Drakos SG, Kfoury AG, Stehlik J, Selzman CH, Reid BB, Terrovitis JV, Li DY. Bridge to Recovery Understanding the Disconnect Between Clinical and Biological Outcomes. Circulation. 2012;126(2):230-241. doi: 10.1161/CIRCULATIONAHA.111.040261

5. Faragallah G, Simaan MA. An Engineering Analysis of the Aortic Valve Dynamics in Patients with Rotary Left Ventricular Assist Devices. J Healthc Eng. 2013;4(3):307-328. doi:10.1260/2040-2295.4.3.307

6. Ferrari M, Kadipasaoglu KA, Croitoru M, Conger J, Myers T, Gregoric I, et al. Evaluation of myocardial function in patients with end-stage heart failure during support with the Jarvik 2000 left ventricular assist device. J Heart Lung Transplant. 2005;24(2):226-228.

7. Frazier OH, Baldwin AC, Demirozu ZT, Segura AM, Hernandez R, Taegtmeyer $\mathrm{H}$, et al. Ventricular reconditioning and pump explanation in patients supported by continuous-flow left ventricular assist devices. J Heart Lung Transplant. 2015;34(6), 766-772. doi: 10.1016/j. healun.2014.09.015

8. Jacob R, Dierberger B, Kissling G. Functional significance of the FrankStarling mechanism under physiological and pathophysiological conditions. Eur Heart J. 1992;13(suppl_E):7-14.

9. Jakovljevic DG, Yacoub MH, Schueler S, MacGowan GA, Velicki L, Seferovic PM, et al. Left ventricular assist device as a bridge to recovery for patients with advanced heart failure. Journal of the American College of Cardiology. 2017;69(15):1924-1933.

10. Kadakia S, Moore R, Ambur V, Toyoda Y. Current status of the implantable LVAD. Gen Thorac Cardiovasc Surg. 2016;64(9):501-508.

11. Kirklin JK, Naftel DC, Pagani FD, Kormos RL, Stevenson LW, Blume ED, Young JB. Seventh INTERMACS annual report: 15,000 patients and counting. J Heart Lung Transplant. 2015;34(12):1495-1504. doi: 10.1016/j.healun.2015.10.003
12. Levin R, Dolgin M, Fox C, Gorlin R. The Criteria Committee of the New York Heart Association in Nomenclature and criteria for diagnosis of diseases of the heart and great vessels, lww handbooks, 9, 253-256.

13. Little WC, Applegate RJ. Congestive heart failure: systolic and diastolic function. J Cardiothorac Vasc Anesth. 1993;7(4):2-5.

14. Mason DT. Regulation of cardiac performance in clinical heart disease. American Journal of Cardiology. 1973;32(4):437-448. doi:10.1016/ S0002-9149(73)80034-7

15. McConnell PI, Del Rio CL, Kwiatkowski P, Farrar DJ, Sun BC. Assessment of Cardiac Function During Axial-flow Left Ventricular Assist Device Support Using a Left Ventricular Pressure-derived Relationship: Comparison With Pre-load Recruitable Stroke Work. J Heart Lung Transplant. 2007;26(2):159-166.

16. McConnell PI, Sun BC. Pressure-volume analysis during axial flow ventricular assist device support. The Journal of Heart and Lung Transplantation. 2006;25(2):256-257.

17. Mikail P, Crosby JR, Slepian MJ, Smith R, Khalpey Z. LVAD Pulsatility Assesses Cardiac Contractility: In Vitro Model Utilizing the Total Artificial Heart and Mock Circulation. ASAIO J. 2019;65(6):580-586. doi: 10.1097/MAT.0000000000000861

18. Morimont P, Lambermont B, Guiot J, Sato VT, Clotuche C, Goffoy J, et al. Ejection Fraction May Not Reflect Contractility: Example in VenoArterial Extracorporeal Membrane Oxygenation for Heart Failure. ASAIO J. 2018;64(4):e68-e71. doi: 10.1097/MAT.0000000000000661

19. Olsen DB. The history of continuous-flow blood pumps. Artif Organs. 24(6):401-404.

20. Oyama MA. Chapter 38 - Mechanisms of Heart Failure. In DC Silverstein, K. Hopper (Eds.), Small Animal Critical Care Medicine (Second Edition) (pp. 205-209). St. Louis: W.B. Saunders.

21. Parmley WW. Pathophysiology of congestive heart failure. Am J Cardiol. 1985;56(2):7A-11A. doi:10.1016/0002-9149(85)91199-3

22. Pombo JF, Troy BL, Russell R O. Left Ventricular Volumes and Ejection Fraction by Echocardiography. Circulation. 1971;43(4), 480-490. doi:10.1161/01.Cir.43.4.480

23. Pool PE, Braunwald E. Fundamental mechanisms in congestive heart failure. The American Journal of Cardiology. 1968;22(1):7-1.

24. Simaan MA, Ferreira A, Chen S, Antaki JF, Galati DG. A Dynamical State Space Representation and Performance Analysis of a FeedbackControlled Rotary Left Ventricular Assist Device. IEEE Transactions on Control Systems Technology. 2009;17(1):15- 28. doi:10.1109/ TCST.2008.912123

25. Simon MA, Kormos RL, Murali S, Nair P, Heffernan M, Gorcsan J, et al. Myocardial recovery using ventricular assist devices: prevalence, clinical characteristics, and outcomes. Circulation. 2005;112(9 suppl):132-136.

26. Stergiopulos N, Meister JJ, Westerhof N. Determinants of stroke volume and systolic and diastolic aortic pressure. Am J Physiol. 1996;270(6):H2050-H2059.

27. Stevenson LW, Rose EA. Left ventricular assist devices: bridges to transplantation, recovery, and destination for whom? Circulation. 2003;108(25):3059-3063.

28. Suga H, Sagawa K. Instantaneous Pressure-Volume Relationships and Their Ratio in the Excised, Supported Canine Left Ventricle. Circulation Research. 1974;35(1):117-126. doi:10.1161/01.res.35.1.117 
29. Symptom and Activity Level Assessment, Heart Failure, American College of Cardiology Clinical Toolkit, 2014.

30. Vasudevan VS, Simaan MA. A system of systems approach to patient treatment with the Left Ventricular Assist Device. Paper presented at the 2017 12th System of Systems Engineering Conference (SoSE).

31. Widmaier EP, Raff H, Strang KT. Vander's human physiology: the mechanisms of body function: McGraw-Hill Higher Education. 2010. 32. Wijayasiri L, Rhodes A, Cecconi M. Cardiac Contractility In J.-L. Vincent \& J. B. Hall (Eds.), Encyclopedia of Intensive Care Medicine (pp. 460462). Berlin, Heidelberg: Springer Berlin Heidelberg.

33. Yancy CW, Jessup M, Bozkurt B, Butler J, Casey DE, Drazner MH, et al. 2013 ACCF/AHA guideline for the management of heart failure: a report of the American College of Cardiology Foundation/American Heart Association Task Force on Practice Guidelines. J Am Coll Cardiol. 